\title{
A propósito de um caso de estridor no Serviço de Urgência
}

\section{A case report of stridor in the Emergency Department}

\begin{abstract}
RESUMO
0 bócio mergulhante é uma patologia relativamente rara e estima-se que corresponda a $7 \%$ de todos os casos de bócio. Na maioria dos doentes é assintomático, mas pode produzir sintomas decorrentes da compressão de estruturas contíguas.

Os autores apresentam o caso de uma doente de 89 anos, com antecedentes pessoais de bócio, admitida no Serviço de Urgência (sala de emergência) por quadro de dificuldade respiratória com estridor. A avaliação efetuada foi compatível com volumoso bócio mergulhante com desvio e deformidade da traqueia, apresentando avaliação analítica a revelar hipertiroidismo. 0 caso foi discutido com a equipa de Cirurgia Endocrinológica, tendo a doente sido submetida a tiroidectomia total.

Com este caso salienta-se a importância da rápida integração dos dados da anamnese em conjunto com o exame objetivo na abordagem a um doente na sala de emergência.

Palabras clave: Estridor, bocio subesternal, hipertiroidismo
\end{abstract}

\section{ABSTRACT}

Substernal goiter is a relatively rare condition and is estimated to correspond to $7 \%$ of all cases of goiter. In most cases it is asymptomatic, but may produce symptons resulting from the compression of contiguous structures.

The authors present the case of a 89 year-old woman, with personal history of goiter, admitted in the Emergency Department (emergency room) due to respiratory distress with stridor. The evaluation was compatible with large substernal goiter with deviation and tracheal deformity, presenting an analytical evaluation revealing with hyperthyroidism. The case was discussed with Endocrinologic Surgery medical team and the patient was submitted to total thyroidectomy.

This case emphasizes the importance of the rapid integration of data from anamnesis and examination in the approach to a patient in the emergency room.

Keywords: Stridor, substernal goiter, hyperthyroidism

\section{CASO CLÍNICO}

Os autores apresentam o caso de uma doente de 89 anos, com antecedentes pessoais de hipertensão arterial, diabetes mellitus tipo 2 e bócio, que recorreu ao Serviço de Urgência por quadro de dificuldade respiratória. Ao exame objetivo na admissão hospitalar (na sala de emergência) apresentava estridor (resolvido após terapêutica médica), tumefação cervical compatível com bócio e alterações à auscultação pulmonar sugestivas de infeção respiratória. Dos exames complementares realizados salienta-se: análises com elevação dos parâmetros inflamatórios e padrão de hipertiroidismo, radiografia torácica com alterações compatíveis com pneumonia bilateral e tomografia computorizada cervical e torácica que evidenciou volumoso bócio multinodular mergulhante com desvio e deformidade da traqueia, bem como das estruturas vasculares à esquerda (Fig. $1 \mathrm{e}$ 2). Admitindo-se ser esta a causa do estridor, o caso foi discutido com a equipa de Cirurgia Endocrinológica, tendo a doente sido submetida a tiroidectomia total. Sem novos
Figura 1. Volumoso bócio multinodular mergulhante (corte coronal de imagem de tomografia computorizada)

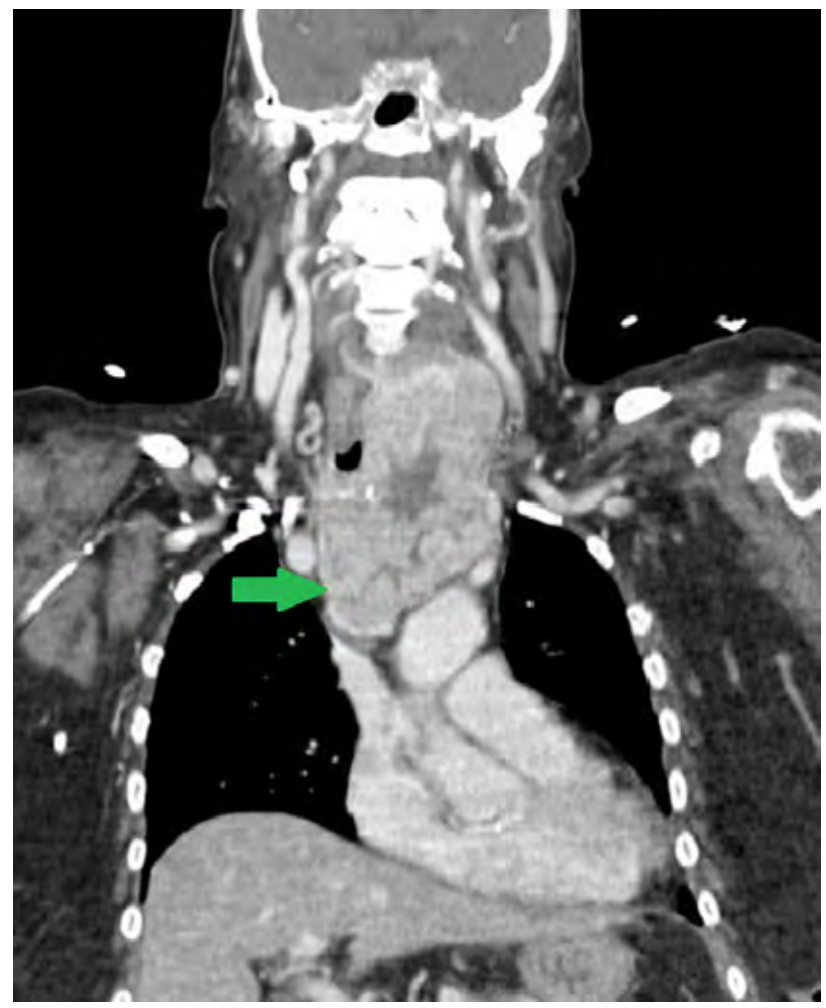

Figura 2. Desvio da traqueia para a direita, condicionado por volumoso bócio mergulhante (corte coronal de imagem de tomografia computorizada)

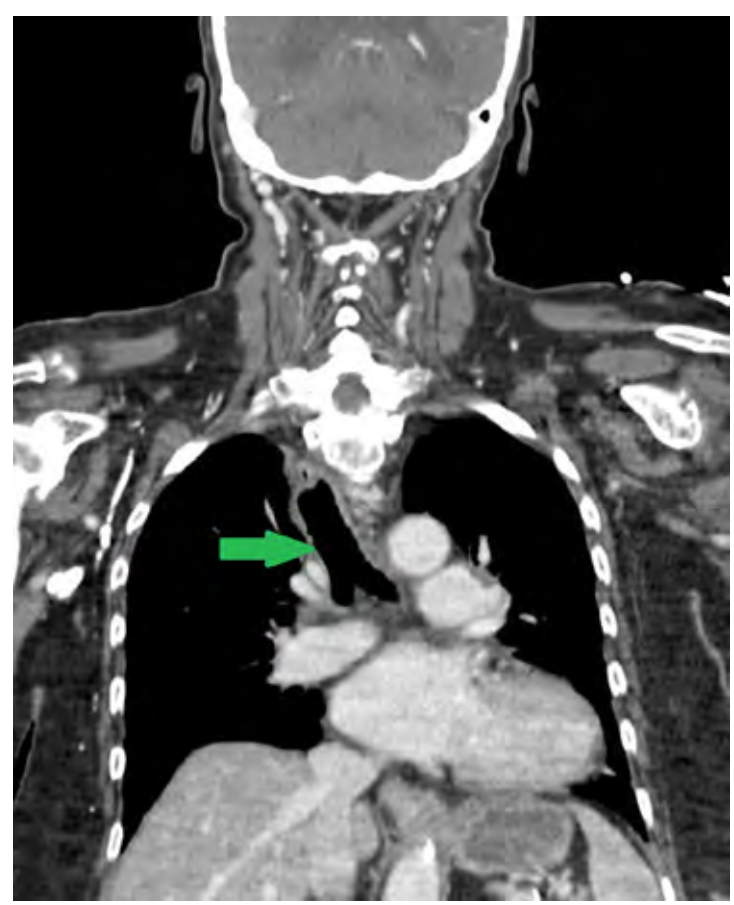


episódios de estridor descritos após a intervenção cirúrgica. 0 bócio mergulhante é uma patologia relativamente rara e estima-se que corresponda a cerca de $7 \%$ de todos os casos de bócio., ${ }^{1,2}$ Na maioria dos doentes é assintomático, mas pode produzir sintomas decorrentes da compressão de estruturas contíguas, ${ }^{3,4}$ como no caso apresentado em que se manifestou por compressão da traqueia. A terapêutica recomendada é cirúrgica, estando preconizada a excisão total ou subtotal da glândula tiroideia. ${ }^{3,4}$

\section{REFERÊNCIAS BIBLIOGRÁFICAS}

1. Huins $\mathrm{CH}$, Georgalas $\mathrm{C}$, Mehrzad H, Tolley NS. A new classification system for retrosternal goitre based on a systematic review of its complications and management. Int J Surg. 2008; 6(1):71-76

2. Naveena S, Chauhana S, Raghavanb N, Chandrashekhara NH, Srikrishnac SV, Dhadedd SB et al. A Large Superior Mediastinal Mass: "Terrible Thyroid Cancer". J Endocrinol Metab. 2016; 6(1):27-29

3. Maia FF, Araúijo LR. Bócio Mergulhante - Quando Operar? Arq Bras Endocrinol Metab. 2002; 46(6): 708-715

4. Chen AY, Bernet VJ, Carty SE, Davies TF, Ganly I, Inabnet WB 3rd et al. American Thyroid Association Statement on Optimal Surgical Management of Goiter. Thyroid. 2014; 24(2):181-189

\section{DIAGNÓSTICO \\ Bócio mergulhante}

\section{Sónia Martins, Ana Sanches}

Serviço de Medicina V Centro Hospitalar do Médio Tejo (CHMT) 